\title{
The charge asymmetry in superconductivity of hole- and electron-doped cuprates
}

\author{
Tianxing Ma and Shiping Feng \\ Department of Physics, Beijing Normal University, Beijing 100875, China
}

\begin{abstract}
Within the $t-t^{\prime}-J$ model, the charge asymmetry in superconductivity of hole- and electron-doped cuprates is studied based on the kinetic energy driven superconducting mechanism. It is shown that superconductivity appears over a narrow range of doping in electron-doped cuprates, and the superconducting transition temperature displays the same kind of the doping controlled behavior that is observed in the hole-doped case. However, the maximum achievable superconducting transition temperature in the optimal doping in electron-doped cuprates is much lower than that of the hole-doped case due to the electron-hole asymmetry.
\end{abstract}

74.20.Mn, 74.62.Dh, 74.25.Dw

The parent compounds of cuprate superconductors are believed to belong to a class of materials known as Mott insulators with the antiferromagnetic (AF) long-range order (AFLRO), then superconductivity occurs by the electron or hole doping ${ }^{1-4}$. Both hole-doped and electrondoped cuprate superconductors have the layered structure of the square lattice of the $\mathrm{CuO}_{2}$ plane separated by insulating layers ${ }^{1,4}$. It has been found from experiments that only an approximate symmetry in the phase diagram exists about the zero doping line between electronand hole-doped cuprates ${ }^{5,6}$. For hole-doped cuprates ${ }^{1-3}$, AFLRO disappears rapidly with doping, and is replaced by a disordered spin liquid phase, then the systems become superconducting (SC) over a wide range of the hole doping concentration $\delta$, around the optimal $\delta \sim 0.15$, however, AFLRO survives until superconductivity appears over a narrow range of $\delta$ around the optimal $\delta \sim 0.15$ in electron-doped cuprates, where the maximum achievable $\mathrm{SC}$ transition temperature is much lower than hole-doped cuprates ${ }^{4,7,8}$. Although this electronhole asymmetry is observed in the phase diagram ${ }^{1,5,6}$, the charge carrier Cooper pairs in the both optimally electron- and hole-doped cuprate superconductors have a dominated d-wave symmetry ${ }^{9-12}$. Since the strong electron correlation is common for both hole-doped and electron-doped cuprates, many of the physical properties of electron-doped cuprates resemble that of the holedoped case. By virtue of systematic studies using the nuclear magnetic resonance, and muon spin rotation techniques, particularly the inelastic neutron scattering, it has been well established that the AF short-range correlation in both hole- and electron-doped cuprate superconductors coexists with the SC state $\mathrm{e}^{1,13-15}$. These provide a clear link between the charge carrier pairing mechanism and magnetic excitations, and show that both holeand electron-doped cuprate superconductors have similar underlying SC mechanism ${ }^{16}$.

Within the $t-J$ model, we $\mathrm{e}^{17,18}$ have discussed the kinetic energy driven SC mechanism in hole-doped cuprates based on the charge-spin separation (CSS) fermion-spin theory ${ }^{19}$, where the dressed holons interact occurring directly through the kinetic energy by exchanging dressed spin excitations, leading to a net attractive force between dressed holons, then the electron Cooper pairs originating from the dressed holon pairing state are due to the charge-spin recombination, and their condensation reveals the SC ground-state ${ }^{17}$. The SC transition temperature is controlled by both charge carrier gap function and single particle coherent weight. This single particle coherent weight grows linearly with increasing doping in the underdoped and optimally doped regimes, and then decreases with increasing doping in the overdoped regime, which leads to that the maximal superconducting transition temperature occurs around the optimal doping, and then decreases in both underdoped and overdoped regimes $^{18}$. In this paper, we study the charge asymmetry in superconductivity of hole- and electrondoped cuprates along with this line. We show that superconductivity appears over a narrow range of doping in electron-doped cuprates, and the maximum achievable $\mathrm{SC}$ transition temperature in the optimal doping is lower than that of the hole-doped case due to the electron-hole asymmetry.

In both hole- and electron-doped cuprates, the characteristic feature is the presence of the two-dimensional $\mathrm{CuO}_{2}$ plane ${ }^{1,4}$ as mentioned above, and it seems evident that the unusual behaviors are dominated by this plane. Although the $t-J$ model captures the essential physics of the doped $\mathrm{CuO}_{2}$ plane ${ }^{20}$, the electron-hole asymmetry may be accounted for by including further neighbor hoping $t^{\prime 21}$. Therefore we start from the $t-t^{\prime}-J$ model,

$$
\begin{aligned}
H & =-t \sum_{i \hat{\eta} \sigma} C_{i \sigma}^{\dagger} C_{i+\hat{\eta} \sigma}+t^{\prime} \sum_{i \hat{\tau} \sigma} C_{i \sigma}^{\dagger} C_{i+\hat{\tau} \sigma} \\
& +\mu \sum_{i \sigma} C_{i \sigma}^{\dagger} C_{i \sigma}+J \sum_{i \hat{\eta}} \mathbf{S}_{i} \cdot \mathbf{S}_{i+\hat{\eta}},
\end{aligned}
$$

with $\hat{\eta}= \pm \hat{x}, \pm \hat{y}, \hat{\tau}= \pm \hat{x} \pm \hat{y}, C_{i \sigma}^{\dagger}\left(C_{i \sigma}\right)$ is the electron creation (annihilation) operator, $\mathbf{S}_{i}=C_{i}^{\dagger} \vec{\sigma} C_{i} / 2$ is spin operator with $\vec{\sigma}=\left(\sigma_{x}, \sigma_{y}, \sigma_{z}\right)$ as Pauli matrices, and $\mu$ is the chemical potential. For the electron doping, we can perform a particle-hole transformation $C_{i \sigma} \rightarrow C_{i-\sigma}^{\dagger}$, so that the difference between hole and electron doping is expressed as the sign difference of the hopping parameters, i.e., $t>0$ and $t^{\prime}>0$ for hole doping and $t<0$ and $t^{\prime}<0$ for electron doping ${ }^{22}$, then the $t-t^{\prime}-J$ model (1) in both hole- and electron-doped cases is always subject to an important on-site local constraint to avoid the double 
occupancy, i.e., $\sum_{\sigma} C_{i \sigma}^{\dagger} C_{i \sigma} \leq 1$. This single occupancy local constraint can be treated properly within the CSS fermion-spin theory ${ }^{19}$, where the constrained electron operators are decoupled as, $C_{i \uparrow}=h_{i \uparrow}^{\dagger} S_{i}^{-}, C_{i \downarrow}=h_{i \downarrow}^{\dagger} S_{i}^{+}$, with the spinful fermion operator $h_{i \sigma}=e^{-i \Phi_{i \sigma}} h_{i}$ describes the charge degree of freedom together with some effects of the spin configuration rearrangements due to the presence of the doped charge carrier itself (dressed charge carrier), while the spin operator $S_{i}$ describes the spin degree of freedom (dressed spin), then the electron local constraint for the single occupancy is satisfied in analytical calculations, and low-energy behavior of the $t-t^{\prime}-J$ model (1) in this CSS fermion-spin representation can be expressed as ${ }^{17-19}$,

$$
\begin{aligned}
H & =-t \sum_{i \hat{\eta}}\left(h_{i \uparrow} S_{i}^{+} h_{i+\hat{\eta} \uparrow}^{\dagger} S_{i+\hat{\eta}}^{-}+h_{i \downarrow} S_{i}^{-} h_{i+\hat{\eta} \downarrow}^{\dagger} S_{i+\hat{\eta}}^{+}\right) \\
& +t^{\prime} \sum_{i \hat{\tau}}\left(h_{i \uparrow} S_{i}^{+} h_{i+\hat{\tau} \uparrow}^{\dagger} S_{i+\hat{\tau}}^{-}+h_{i \downarrow} S_{i}^{-} h_{i+\hat{\tau} \downarrow}^{\dagger} S_{i+\hat{\tau}}^{+}\right) \\
& -\mu \sum_{i \sigma} h_{i \sigma}^{\dagger} h_{i \sigma}+J_{\mathrm{eff}} \sum_{i \hat{\eta}} \mathbf{S}_{i} \cdot \mathbf{S}_{i+\hat{\eta}},
\end{aligned}
$$

with $J_{\text {eff }}=(1-x)^{2} J$, and $\delta=\left\langle h_{i \sigma}^{\dagger} h_{i \sigma}\right\rangle=\left\langle h_{i}^{\dagger} h_{i}\right\rangle$ is the doping concentration. As a consequence, the kinetic energy terms in the $t-t^{\prime}-J$ model have been expressed as the interactions between dressed charge carriers and spins, which reflects that even the kinetic energy terms in the $t-t^{\prime}-J$ Hamiltonian have strong Coulombic contributions due to the restriction of no doubly occupancy of a given site. These interactions between dressed charge carriers and spins are quite strong, and we $\mathrm{e}^{17,18}$ have shown that in the case without AFLRO, these interactions can induce the dressed charge carrier pairing state (then the electron Cooper pairing state) by exchanging dressed spin excitations in the higher power of the doping concentration $\delta$. Since the SC state in both hole- and electron-doped cuprates is characterized by the electron Cooper pairs, forming SC quasiparticles ${ }^{9,12}$, and in the real space the gap function and pairing force have a range of one lattice spacing ${ }^{23}$, therefore the order parameter for the electron Cooper pair can be expressed as, $\Delta=\left\langle C_{i \uparrow}^{\dagger} C_{i+\hat{\eta} \downarrow}^{\dagger}-\right.$ $\left.C_{i \downarrow}^{\dagger} C_{i+\hat{\eta} \uparrow}^{\dagger}\right\rangle=\left\langle h_{i \uparrow} h_{i+\hat{\eta} \downarrow} S_{i}^{+} S_{i+\hat{\eta}}^{-}-h_{i \downarrow} h_{i+\hat{\eta} \uparrow} S_{i}^{-} S_{i+\hat{\eta}}^{+}\right\rangle=$ $-\left\langle S_{i}^{+} S_{i+\hat{\eta}}^{-}\right\rangle \Delta_{h}$, with the dressed charge carrier pairing order parameter $\Delta_{h}=\left\langle h_{i+\hat{\eta} \downarrow} h_{i \uparrow}-h_{i+\hat{\eta} \uparrow} h_{i \downarrow}\right\rangle$, which shows that the SC order parameter is closely related to the dressed charge carrier pairing amplitude, and is proportional to the number of charge carriers, and not to the number of electrons. Following the Eliashberg's strong coupling theory ${ }^{24}$, we obtain the self-consistent equations that satisfied by the full dressed charge carrier diagonal and off-diagonal Green's functions as ${ }^{17}$,

$$
\begin{aligned}
g(k) & =g^{(0)}(k)+g^{(0)}(k)\left[\Sigma_{1}^{(h)}(k) g(k)\right. \\
& \left.-\Sigma_{2}^{(h)}(-k) \Im^{\dagger}(k)\right], \\
\Im^{\dagger}(k) & =g^{(0)}(-k)\left[\Sigma_{1}^{(h)}(-k) \Im^{\dagger}(-k)\right.
\end{aligned}
$$

$$
\left.+\Sigma_{2}^{(h)}(-k) g(k)\right]
$$

respectively, where the four-vector notation $k=\left(\mathbf{k}, i \omega_{n}\right)$, the dressed charge carrier mean-field (MF) diagonal Green's function ${ }^{17} g^{(0)-1}(k)=i \omega_{n}-\xi_{\mathbf{k}}$, the MF dressed charge carrier excitation spectrum $\xi_{\mathrm{k}}=$ $Z t \chi_{1} \gamma_{\mathbf{k}}-Z t^{\prime} \chi_{2} \gamma_{\mathbf{k}}^{\prime}-\mu$, with $\gamma_{\mathbf{k}}=(1 / Z) \sum_{\hat{\eta}} e^{i \mathbf{k} \cdot \hat{\eta}}, \gamma_{\mathbf{k}}^{\prime}=$ $(1 / Z) \sum_{\hat{\tau}} e^{i \mathbf{k} \cdot \hat{\tau}}, Z$ is the number of the nearest neighbor or second-nearest neighbour sites, the dressed spin correlation functions $\chi_{1}=\left\langle S_{i}^{+} S_{i+\hat{\eta}}^{-}\right\rangle$and $\chi_{2}=\left\langle S_{i}^{+} S_{i+\hat{\tau}}^{-}\right\rangle$, and the dressed charge carrier self-energy functions ${ }^{17,18}$,

$$
\begin{aligned}
\Sigma_{1}^{(h)}(k) & =\frac{1}{N^{2}} \sum_{\mathbf{p}, \mathbf{p}^{\prime}}\left(Z t \gamma_{\mathbf{p}+\mathbf{p}^{\prime}+\mathbf{k}}-Z t^{\prime} \gamma_{\mathbf{p}+\mathbf{p}^{\prime}+\mathbf{k}}^{\prime}\right)^{2} \\
& \times \frac{1}{\beta} \sum_{i p_{m}} g(p+k) \frac{1}{\beta} \sum_{i p_{m}^{\prime}} D^{(0)}\left(p^{\prime}\right) D^{(0)}\left(p^{\prime}+p\right), \\
\Sigma_{2}^{(h)}(k) & =\frac{1}{N^{2}} \sum_{\mathbf{p}, \mathbf{p}^{\prime}}\left(Z t \gamma_{\mathbf{p}+\mathbf{p}^{\prime}+\mathbf{k}}-Z t^{\prime} \gamma_{\mathbf{p}+\mathbf{p}^{\prime}+\mathbf{k}}^{\prime}\right)^{2} \\
& \times \frac{1}{\beta} \sum_{i p_{m}} \Im(-p-k) \frac{1}{\beta} \sum_{i p_{m}^{\prime}} D^{(0)}\left(p^{\prime}\right) D^{(0)}\left(p^{\prime}+p\right),
\end{aligned}
$$

where $p=\left(\mathbf{p}, i p_{m}\right), p^{\prime}=\left(\mathbf{p}^{\prime}, i p_{m}^{\prime}\right)$, and the MF dressed spin Green's function ${ }^{17}$,

$$
D^{(0)}(p)=\frac{B_{\mathbf{p}}}{\left(i p_{m}\right)^{2}-\omega_{\mathbf{p}}^{2}},
$$

with $B_{\mathbf{p}}=\lambda_{1}\left[2 \chi_{1}^{z}\left(\epsilon \gamma_{\mathbf{p}}-1\right)+\chi_{1}\left(\gamma_{\mathbf{p}}-\epsilon\right)\right]-\lambda_{2}\left(2 \chi_{2}^{z} \gamma_{\mathbf{p}}^{\prime}-\right.$ $\left.\chi_{2}\right), \lambda_{1}=2 Z J_{\text {eff }}, \lambda_{2}=4 Z \phi_{2} t^{\prime}, \epsilon=1+2 t \phi_{1} / J_{\text {eff }}$, and the MF dressed spin excitation spectrum $\omega_{\mathbf{p}}^{2}=$ $A_{1}\left(\gamma_{\mathbf{k}}\right)^{2}+A_{2}\left(\gamma_{\mathbf{k}}^{\prime}\right)^{2}+A_{3} \gamma_{\mathbf{k}} \gamma_{\mathbf{k}}^{\prime}+A_{4} \gamma_{\mathbf{k}}+A_{5} \gamma_{\mathbf{k}}^{\prime}+A_{6}$, with $A_{1}=\alpha \epsilon \lambda_{1}^{2}\left(\epsilon \chi_{1}^{z}+\chi_{1} / 2\right), A_{2}=\alpha \lambda_{2}^{2} \chi_{2}^{z}, A_{3}=$ $-\alpha \lambda_{1} \lambda_{2}\left(\epsilon \chi_{1}^{z}+\epsilon \chi_{2}^{z}+\chi_{1} / 2\right), A_{4}=-\epsilon \lambda_{1}^{2}\left[\alpha\left(\chi_{1}^{z}+\epsilon \chi_{1} / 2\right)+\right.$ $\left(\alpha C_{1}^{z}+(1-\alpha) /(4 Z)-\alpha \epsilon \chi_{1} /(2 Z)\right)+\left(\alpha C_{1}+(1-\right.$ $\left.\left.\alpha) /(2 Z)-\alpha \chi_{1}^{z} / 2\right) / 2\right]+\alpha \lambda_{1} \lambda_{2}\left(C_{3}+\epsilon \chi_{2}\right) / 2, \quad A_{5}=$ $-3 \alpha \lambda_{2}^{2} \chi_{2} /(2 Z)+\alpha \lambda_{1} \lambda_{2}\left(\chi_{1}^{z}+\epsilon \chi_{1} / 2+C_{3}^{z}\right), A_{6}=\lambda_{1}^{2}\left[\alpha C_{1}^{z}+\right.$ $(1-\alpha) /(4 Z)-\alpha \epsilon \chi_{1} /(2 Z)+\epsilon^{2}\left(\alpha C_{1}+(1-\alpha) /(2 Z)-\right.$ $\left.\left.\left.\alpha \chi_{1}^{z} / 2\right) / 2\right]+\lambda_{2}^{2}\left(\alpha C_{2}+(1-\alpha) /(2 Z)-\alpha \chi_{2}^{z} / 2\right) / 2\right)-$ $\alpha \in \lambda_{1} \lambda_{2} C_{3}$, and the dressed charge carrier's particlehole parameters $\phi_{1}=\left\langle h_{i \sigma}^{\dagger} h_{i+\hat{\eta} \sigma}\right\rangle, \phi_{2}=\left\langle h_{i \sigma}^{\dagger} h_{i+\hat{\tau} \sigma}\right\rangle$, the dressed spin correlation functions $\chi_{1}^{z}=\left\langle S_{i}^{z} S_{i+\hat{\eta}}^{z}\right\rangle$, $\chi_{2}^{z}=\left\langle S_{i}^{z} S_{i+\hat{\tau}}^{z}\right\rangle, C_{1}=\left(1 / Z^{2}\right) \sum_{\hat{\eta}, \hat{\eta}^{\prime}}\left\langle S_{i+\hat{\eta}^{\prime}}^{+} S_{i+\hat{\eta}^{\prime}}^{-}\right\rangle, C_{1}^{z}=$ $\left(1 / Z^{2}\right) \sum_{\hat{\eta}, \hat{\eta}^{\prime}}\left\langle S_{i+\hat{\eta}}^{z} S_{i+\hat{\eta}^{\prime}}^{z}\right\rangle, C_{2}=\left(1 / Z^{2}\right) \sum_{\hat{\tau}, \hat{\tau}^{\prime}}\left\langle S_{i+\hat{\tau}}^{+} S_{i+\hat{\tau}^{\prime}}^{-}\right\rangle$, $C_{3}=(1 / Z) \sum_{\hat{\tau}}\left\langle S_{i+\hat{\eta}}^{+} S_{i+\hat{\tau}}^{-}\right\rangle, \quad$ and $C_{3}^{z}=(1 / Z) \sum_{\hat{\tau}}\left\langle S_{i+\hat{\eta}}^{z} S_{i+\hat{\tau}}^{z}\right\rangle$. In order to satisfy the sum rule of the correlation function $\left\langle S_{i}^{+} S_{i}^{-}\right\rangle=1 / 2$ in the case without AFLRO, the important decoupling parameter $\alpha$ has been introduced in the MF calculation ${ }^{17}$, which can be regarded as the vertex correction.

In Eq. (4), the self-energy function $\Sigma_{2}^{(h)}(k)$ is called as the effective dressed charge carrier gap function since it contains both pairing force and dressed charge carrier gap function, while the self-energy function $\Sigma_{1}^{(h)}(k)$ renormalizes the MF dressed charge carrier spectrum, and there- 
fore it describes the single particle (quasiparticle) coherence. In particular, $\Sigma_{2}^{(h)}(k)$ is an even function of $i \omega_{n}$, while $\Sigma_{1}^{(h)}(k)$ is not. For the convenience of discussions, we separate $\Sigma_{1}^{(h)}(k)$ into its symmetric and antisymmetric parts as, $\Sigma_{1}^{(h)}(k)=\Sigma_{1 e}^{(h)}(k)+i \omega_{n} \Sigma_{1 o}^{(h)}(k)$, then $\Sigma_{1 e}^{(h)}(k)$ and $\Sigma_{1 o}^{(h)}(k)$ are both even functions of $i \omega_{n}$. According to the Eliashberg's strong coupling theory ${ }^{24}$, we can define the charge carrier single particle (quasiparticle) coherent weight $Z_{F}^{-1}(k)=1-\Sigma_{1 o}^{(h)}(k)$. On the other hand, the retarded function $\operatorname{Re} \Sigma_{1 e}^{(h)}(k)$ may be a constant, independent of $(\mathbf{k}, \omega)$. It just renormalizes the chemical potential, and therefore can be neglected ${ }^{24}$. Furthermore, we only study the static limit of the effective dressed charge carrier gap function and single particle coherent weight, i.e., $\Sigma_{2}^{(h)}(k)=\bar{\Delta}_{h}(\mathbf{k})$, and $Z_{F}^{-1}(\mathbf{k})=1-\Sigma_{1 o}^{(h)}(\mathbf{k})$. Although $Z_{F}(\mathbf{k})$ still is a function of $\mathbf{k}$, the wave vector dependence is unimportant, since everything happens at the electron Fermi surface. As in the previous discussions within the $t-J$ model $^{18}$, the special wave vector can be estimated qualitatively from the electron momentum distribution as $\mathbf{k}_{0}=\mathbf{k}_{\mathbf{A}}-\mathbf{k}_{\mathbf{F}}$ with $\mathbf{k}_{\mathbf{A}}=[\pi, \pi]$ and $\mathbf{k}_{\mathbf{F}} \approx[(1-x) \pi / 2,(1-x) \pi / 2]$, which guarantees $Z_{F}=Z_{F}\left(\mathbf{k}_{0}\right)$ near the electron Fermi surface. In this case, the dressed charge carrier diagonal and off-diagonal Green's functions in Eq. (3) can be rewritten explicitly as,

$$
\begin{aligned}
g(k) & =\frac{1}{2}\left(1+\frac{\overline{\xi_{\mathbf{k}}}}{E_{\mathbf{k}}}\right) \frac{Z_{F}}{i \omega_{n}-E_{\mathbf{k}}} \\
& +\frac{1}{2}\left(1-\frac{\overline{\xi_{\mathbf{k}}}}{E_{\mathbf{k}}}\right) \frac{Z_{F}}{i \omega_{n}+E_{\mathbf{k}}}, \\
\Im^{\dagger}(k) & =-\frac{1}{2} \frac{\bar{\Delta}_{h Z}(\mathbf{k})}{E_{\mathbf{k}}}\left(\frac{Z_{F}}{i \omega_{n}-E_{\mathbf{k}}}-\frac{Z_{F}}{i \omega_{n}+E_{\mathbf{k}}}\right),
\end{aligned}
$$

with $\overline{\xi_{\mathbf{k}}}=Z_{F} \xi_{\mathbf{k}}, \bar{\Delta}_{h Z}(\mathbf{k})=Z_{F} \bar{\Delta}_{h}(\mathbf{k})$, and the dressed charge carrier quasiparticle spectrum $E_{\mathbf{k}}=$ $\sqrt{\bar{\xi}_{\mathbf{k}}^{2}+\left|\bar{\Delta}_{h Z}(\mathbf{k})\right|^{2}}$.

Although superconductivity with both d-wave and swave symmetries appear within the $t-J$ model, the SC state has a dominated d-wave symmetry in the optimal doping ${ }^{18}$. Moreover, we ${ }^{25}$ have discussed the effect of the additional second neighbor hopping $t^{\prime}$ on superconductivity, and found that the d-wave SC pairing correlation is enhanced, while the s-wave SC pairing correlation is heavily suppressed. In this paper, we are interested in the charge asymmetry in superconductivity of hole- and electron-doped cuprates. To make the discussions simpler, we only consider the d-wave case, i.e., $\bar{\Delta}_{h Z}(\mathbf{k})=\bar{\Delta}_{h Z} \gamma_{\mathbf{k}}^{(d)}$, with $\gamma_{\mathbf{k}}^{(d)}=\left(\cos k_{x}-\cos k_{y}\right) / 2$. In this case, the dressed charge carrier effective gap parameter and single particle coherent weight in Eq. (4) satisfy the following equations ${ }^{17,18}$,

$$
1=\frac{1}{N^{3}} \sum_{\mathbf{k}, \mathbf{q}, \mathbf{p}}\left(Z t \gamma_{\mathbf{k}+\mathbf{q}}-Z t^{\prime} \gamma_{\mathbf{k}+\mathbf{q}}^{\prime}\right)^{2} \gamma_{\mathbf{k}-\mathbf{p}+\mathbf{q}}^{(d)} \gamma_{\mathbf{k}}^{(d)} \frac{Z_{F}^{2}}{E_{\mathbf{k}}} \frac{B_{\mathbf{q}} B_{\mathbf{p}}}{\omega_{\mathbf{q}} \omega_{\mathbf{p}}}
$$

$$
\begin{aligned}
& \times\left(\frac{F_{1}^{(1)}(\mathbf{k}, \mathbf{q}, \mathbf{p})}{\left(\omega_{\mathbf{p}}-\omega_{\mathbf{q}}\right)^{2}-E_{\mathbf{k}}^{2}}+\frac{F_{1}^{(2)}(\mathbf{k}, \mathbf{q}, \mathbf{p})}{\left(\omega_{\mathbf{p}}+\omega_{\mathbf{q}}\right)^{2}-E_{\mathbf{k}}^{2}}\right) \\
Z_{F} & =1+\frac{1}{N^{2}} \sum_{\mathbf{q}, \mathbf{p}}\left(Z t \gamma_{\mathbf{p}+\mathbf{k}_{\mathbf{0}}}-Z t^{\prime} \gamma_{\mathbf{p}+\mathbf{k}_{\mathbf{0}}}^{\prime}\right)^{2} Z_{F} \frac{B_{\mathbf{q}} B_{\mathbf{p}}}{4 \omega_{\mathbf{q}} \omega_{\mathbf{p}}} \\
& \times\left(\frac{F_{2}^{(1)}(\mathbf{q}, \mathbf{p})}{\left(\omega_{\mathbf{p}}-\omega_{\mathbf{q}}-E_{\left.\mathbf{p}-\mathbf{q}+\mathbf{k}_{\mathbf{0}}\right)^{2}}\right.}+\frac{F_{2}^{(2)}(\mathbf{q}, \mathbf{p})}{\left(\omega_{\mathbf{p}}-\omega_{\mathbf{q}}+E_{\mathbf{p}-\mathbf{q}+\mathbf{k}_{\mathbf{o}}}\right)^{2}}\right. \\
& \left.+\frac{F_{2}^{(3)}(\mathbf{q}, \mathbf{p})}{\left(\omega_{\mathbf{p}}+\omega_{\mathbf{q}}-E_{\mathbf{p}-\mathbf{q}+\mathbf{k}_{\mathbf{0}}}\right)^{2}}+\frac{F_{2}^{(4)}(\mathbf{q}, \mathbf{p})}{\left(\omega_{\mathbf{p}}+\omega_{\mathbf{q}}+E_{\mathbf{p}-\mathbf{q}+\mathbf{k}_{\mathbf{0}}}\right)^{2}}\right)
\end{aligned}
$$

respectively,

where $F_{1}^{(1)}(\mathbf{k}, \mathbf{q}, \mathbf{p})=\left(\omega_{\mathbf{p}}-\omega_{\mathbf{q}}\right)\left[n_{B}\left(\omega_{\mathbf{q}}\right)-n_{B}\left(\omega_{\mathbf{p}}\right)\right][1-$ $\left.2 n_{F}\left(E_{\mathbf{k}}\right)\right]+E_{\mathbf{k}}\left[n_{B}\left(\omega_{\mathbf{p}}\right) n_{B}\left(-\omega_{\mathbf{q}}\right)+n_{B}\left(\omega_{\mathbf{q}}\right) n_{B}\left(-\omega_{\mathbf{p}}\right)\right]$, $F_{1}^{(2)}(\mathbf{k}, \mathbf{q}, \mathbf{p})=-\left(\omega_{\mathbf{p}}+\omega_{\mathbf{q}}\right)\left[n_{B}\left(\omega_{\mathbf{q}}\right)-n_{B}\left(-\omega_{\mathbf{p}}\right)\right][1-$ $\left.2 n_{F}\left(E_{\mathbf{k}}\right)\right]+E_{\mathbf{k}}\left[n_{B}\left(\omega_{\mathbf{p}}\right) n_{B}\left(\omega_{\mathbf{q}}\right)+n_{B}\left(-\omega_{\mathbf{p}}\right) n_{B}\left(-\omega_{\mathbf{q}}\right)\right]$, $F_{2}^{(1)}(\mathbf{q}, \mathbf{p})=n_{F}\left(E_{\mathbf{p}-\mathbf{q}+\mathbf{k}_{\mathbf{0}}}\right)\left[n_{B}\left(\omega_{\mathbf{q}}\right)-n_{B}\left(\omega_{\mathbf{p}}\right)\right]-$ $n_{B}\left(\omega_{\mathbf{p}}\right) n_{B}\left(-\omega_{\mathbf{q}}\right), F_{2}^{(2)}(\mathbf{q}, \mathbf{p})=n_{F}\left(E_{\mathbf{p}-\mathbf{q}+\mathbf{k}_{\mathbf{o}}}\right)\left[n_{B}\left(\omega_{\mathbf{p}}\right)-\right.$ $\left.n_{B}\left(\omega_{\mathbf{q}}\right)\right] \quad-\quad-n_{B}\left(\omega_{\mathbf{q}}\right) n_{B}\left(-\omega_{\mathbf{p}}\right)$, $F_{2}^{(3)}(\mathbf{q}, \mathbf{p})=n_{F}\left(E_{\mathbf{p}-\mathbf{q}+\mathbf{k}_{\mathbf{0}}}\right)\left[n_{B}\left(\omega_{\mathbf{q}}\right)-n_{B}\left(-\omega_{\mathbf{p}}\right)\right]+$ $n_{B}\left(\omega_{\mathbf{p}}\right) n_{B}\left(\omega_{\mathbf{q}}\right), F_{2}^{(4)}(\mathbf{q}, \mathbf{p})=n_{F}\left(E_{\mathbf{p}-\mathbf{q}+\mathbf{k}_{\mathbf{0}}}\right)\left[n_{B}\left(-\omega_{\mathbf{q}}\right)-\right.$ $\left.n_{B}\left(\omega_{\mathbf{p}}\right)\right]+n_{B}\left(-\omega_{\mathbf{p}}\right) n_{B}\left(-\omega_{\mathbf{q}}\right)$, and $n_{B}(\omega)$ and $n_{F}(\omega)$ are the boson and fermion distribution functions, respectively. These two equations must be solved simultaneously with other self-consistent equations ${ }^{17,18}$,

$$
\begin{aligned}
\phi_{1} & =\frac{1}{2 N} \sum_{\mathbf{k}} \gamma_{\mathbf{k}}\left(1-\frac{\xi_{\mathbf{k}}}{E_{\mathbf{k}}} \operatorname{th}\left[\frac{1}{2} \beta E_{\mathbf{k}}\right]\right), \\
\phi_{2} & =\frac{1}{2 N} \sum_{\mathbf{k}} \gamma_{\mathbf{k}}^{\prime}\left(1-\frac{\xi_{\mathbf{k}}}{E_{\mathbf{k}}} \operatorname{th}\left[\frac{1}{2} \beta E_{\mathbf{k}}\right]\right), \\
\delta & =\frac{1}{2 N} \sum_{\mathbf{k}}\left(1-\frac{\xi_{\mathbf{k}}}{E_{\mathbf{k}}} \operatorname{th}\left[\frac{1}{2} \beta E_{\mathbf{k}}\right]\right), \\
\chi_{1} & =\frac{1}{N} \sum_{\mathbf{k}} \gamma_{\mathbf{k}} \frac{B_{\mathbf{k}}}{2 \omega_{\mathbf{k}}} \operatorname{coth}\left[\frac{1}{2} \beta \omega_{\mathbf{k}}\right], \\
\chi_{2} & =\frac{1}{N} \sum_{\mathbf{k}} \gamma_{\mathbf{k}}^{\prime} \frac{B_{\mathbf{k}}}{2 \omega_{\mathbf{k}}} \operatorname{coth}\left[\frac{1}{2} \beta \omega_{\mathbf{k}}\right], \\
C_{1} & =\frac{1}{N} \sum_{\mathbf{k}} \gamma_{\mathbf{k}}^{2} \frac{B_{\mathbf{k}}}{2 \omega_{\mathbf{k}}} \operatorname{coth}\left[\frac{1}{2} \beta \omega_{\mathbf{k}}\right], \\
C_{2} & =\frac{1}{N} \sum_{\mathbf{k}} \gamma_{\mathbf{k}}^{\prime 2} \frac{B_{\mathbf{k}}}{2 \omega_{\mathbf{k}}} \operatorname{coth}\left[\frac{1}{2} \beta \omega_{\mathbf{k}}\right], \\
C_{3} & =\frac{1}{N} \sum_{\mathbf{k}} \gamma_{\mathbf{k}} \gamma_{\mathbf{k}}^{\prime} \frac{B_{\mathbf{k}}}{2 \omega_{\mathbf{k}}} \operatorname{coth}\left[\frac{1}{2} \beta \omega_{\mathbf{k}}\right], \\
\frac{1}{2} & =\frac{1}{N} \sum_{\mathbf{k}} \frac{B_{\mathbf{k}}}{2 \omega_{\mathbf{k}}} \operatorname{coth}\left[\frac{1}{2} \beta \omega_{\mathbf{k}}\right], \\
\chi_{1}^{z} & =\frac{1}{N} \sum_{\mathbf{k}} \gamma_{\mathbf{k}} \frac{B_{z}(\mathbf{k})}{2 \omega_{z}(\mathbf{k})} \operatorname{coth}\left[\frac{1}{2} \beta \omega_{z}(\mathbf{k})\right], \\
\chi_{2}^{z} & =\frac{1}{N} \sum_{\mathbf{k}} \gamma_{\mathbf{k}}^{\prime} \frac{B_{z}(\mathbf{k})}{2 \omega_{z}(\mathbf{k})} \operatorname{coth}\left[\frac{1}{2} \beta \omega_{z}(\mathbf{k})\right], \\
&
\end{aligned}
$$




$$
\begin{aligned}
& C_{1}^{z}=\frac{1}{N} \sum_{\mathbf{k}} \gamma_{\mathbf{k}}^{2} \frac{B_{z}(\mathbf{k})}{2 \omega_{z}(\mathbf{k})} \operatorname{coth}\left[\frac{1}{2} \beta \omega_{z}(\mathbf{k})\right], \\
& C_{3}^{z}=\frac{1}{N} \sum_{\mathbf{k}} \gamma_{\mathbf{k}} \gamma_{\mathbf{k}}^{\prime} \frac{B_{z}(\mathbf{k})}{2 \omega_{z}(\mathbf{k})} \operatorname{coth}\left[\frac{1}{2} \beta \omega_{z}(\mathbf{k})\right],
\end{aligned}
$$

then all the above order parameters, decoupling parameter $\alpha$, and chemical potential $\mu$ are determined by the self-consistent calculation ${ }^{17,18}$.

It has been shown ${ }^{17}$ that the dressed charge carrier pairing state originating from the kinetic energy terms by exchanging dressed spin excitations can lead to form the electron Cooper pairing state, where the SC gap function is obtained from the electron off-diagonal Green's function $\Gamma^{\dagger}\left(i-j, t-t^{\prime}\right)=\left\langle\left\langle C_{i \uparrow}^{\dagger}(t) ; C_{j \downarrow}^{\dagger}\left(t^{\prime}\right)\right\rangle\right\rangle$, which is a convolution of the dressed spin Green's function and dressed charge carrier off-diagonal Green's function, and reflects the charge-spin recombination ${ }^{26}$. In the present case, this electron off-diagonal Green's function can be obtained in terms of the MF dressed spin Green's function (5) and dressed charge carrier off-diagonal Green's function (6b) as,

$$
\begin{aligned}
\Gamma^{\dagger}(k) & =\frac{1}{N} \sum_{\mathbf{p}} \frac{Z_{F} \bar{\Delta}_{h Z}(\mathbf{p}-\mathbf{k})}{E_{\mathbf{p}-\mathbf{k}}} \frac{B_{\mathbf{p}}}{2 \omega_{\mathbf{p}}} \\
& \times\left(\frac{\left(\omega_{\mathbf{p}}+E_{\mathbf{p}-\mathbf{k}}\right)\left[n_{B}\left(\omega_{\mathbf{p}}\right)+n_{F}\left(-E_{\mathbf{p}-\mathbf{k}}\right)\right]}{\left(i \omega_{n}\right)^{2}-\left(\omega_{\mathbf{p}}+E_{\mathbf{p}-\mathbf{k}}\right)^{2}}\right. \\
& \left.-\frac{\left(\omega_{\mathbf{p}}-E_{\mathbf{p}-\mathbf{k}}\right)\left[n_{B}\left(\omega_{\mathbf{p}}\right)+n_{F}\left(E_{\mathbf{p}-\mathbf{k}}\right)\right]}{\left(i \omega_{n}\right)^{2}-\left(\omega_{\mathbf{p}}-E_{\mathbf{p}-\mathbf{k}}\right)^{2}}\right) .
\end{aligned}
$$

With the help of this electron off-diagonal Green's function, the SC gap function is obtained as $\Delta(\mathbf{k})=$ $-(1 / \beta) \sum_{i \omega_{n}} \Gamma^{\dagger}\left(\mathbf{k}, i \omega_{n}\right)$, and can be evaluated explicitly,

$$
\begin{aligned}
\Delta(\mathbf{k}) & =-\frac{1}{N} \sum_{\mathbf{p}} \frac{Z_{F} \bar{\Delta}_{h Z}(\mathbf{p}-\mathbf{k})}{2 E_{\mathbf{p}-\mathbf{k}}} \\
& \times \tanh \left[\frac{1}{2} \beta E_{\mathbf{p}-\mathbf{k}}\right] \frac{B_{\mathbf{p}}}{2 \omega_{\mathbf{p}}} \operatorname{coth}\left[\frac{1}{2} \beta \omega_{\mathbf{p}}\right],
\end{aligned}
$$

which shows that the SC transition temperature $T_{c}$ occurring in the case of the SC gap parameter $\Delta=0$ is identical to the dressed charge carrier pair transition temperature occurring in the case of the effective dressed charge carrier pairing gap parameter $\bar{\Delta}_{h Z}=0$. Since the absolute values of $t$ and $t^{\prime}$ are almost same for both hole- and electron-doped cuprates ${ }^{21}$, and therefore in this paper, the commonly used parameters are chosen as $t / J=2.5$ and $t^{\prime} / t=0.3$ for the hole doping, and $t / J=-2.5$ and $t^{\prime} / t=0.3$ for the electron doping. In Fig. 1, we plot the SC transition temperature $T_{c}$ as a function of the doping concentration $\delta$ for (a) the electron doping and (b) the hole doping in comparison with the corresponding experimental results of $\operatorname{Pr}_{2-x} \mathrm{Ce}_{x} \mathrm{CuO}_{4-y} 8$ and $\mathrm{La}_{2-x} \mathrm{Sr}_{x} \mathrm{CuO}_{4}{ }^{3}$ (inset). Our results indicate that for the hole-doped case, superconductivity appears over a wide range of doping, where the maximal SC transition temperature $\mathrm{T}_{c}$ occurs around the optimal doping concentration $\delta_{\mathrm{opt}} \approx 0.15$, and then decreases in both underdoped and overdoped regimes. In analogy to the phase diagram of the hole-doped case, superconductivity appears over a narrow range of doping in the electron-doped side, where the $\mathrm{SC}$ transition temperature $\mathrm{T}_{c}$ increases sharply with increasing doping in the underdoped regime, and reaches a maximum in the optimal doping $\delta_{\text {opt }} \approx 0.14$, then decreases sharply with increasing doping in the overdoped regime. However, the maximum achievable SC transition temperature in the optimal doping in electron-doped cuprates is much lower than that of the hole-doped case due to the electron-hole asymmetry. Using an reasonably estimative value of $J \sim 800 \mathrm{~K}$ to $1200 \mathrm{~K}$ in doped cuprates, the SC transition temperature in the optimal doping is $\mathrm{T}_{c} \approx 0.22 J \approx 176 \mathrm{~K} \sim 264 \mathrm{~K}$ for the holedoped case, and $\mathrm{T}_{c} \approx 0.136 J \approx 108 \mathrm{~K} \sim 163 \mathrm{~K}$ for the electron-doped case, in qualitative agreement with the corresponding experimental data ${ }^{3,4,8}$.

The essential physics of the doping dependent SC transition temperature in the electron-doped case is almost the same as in the hole-doped side, and detailed explanations have been given in Ref. [18]. In the framework of the kinetic energy driven superconductivity ${ }^{17}$, the self-energy function $\Sigma_{2}^{(h)}(\mathbf{k})$ describes the effective dressed charge carrier pairing gap function, and measures the strength of the binding of charge carrier pairs, while the antisymmetric part of the self-energy function $\Sigma_{1 o}^{(h)}(\mathbf{k})\left(\right.$ then $\left.Z_{F}\right)$ describes the single particle (quasiparticle) coherence, and therefore $Z_{F}$ is closely related to the quasiparticle density. Since the SC state is established through an emerging quasiparticle ${ }^{27}$, then the SC state is controlled by both gap function and quasiparticle coherence, which is reflected explicitly in the selfconsistent equations (7a) and (7b). It has been shown that the doping dependent behavior of the single particle coherent weight resembles that of the superfluid density in doped cuprates ${ }^{18}$, i.e., $Z_{F}$ grows linearly with the doping concentration in the underdoped and optimally doped regimes, and then decreases with increasing doping in the overdoped regime, which leads to that the SC transition temperature reaches a maximum in the optimal doping,

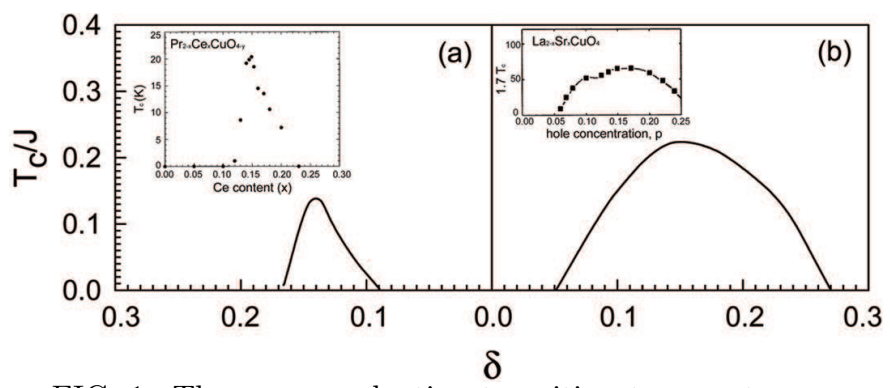

FIG. 1. The superconducting transition temperature as a function of the doping concentration with (a) $t / J=-2.5$ and $t^{\prime} / t=0.3$ for the electron doping and (b) $t / J=2.5$ and $t^{\prime} / t=0.3$ for the hole doping. Inset: the corresponding experimental results of $\mathrm{Pr}_{2-x} \mathrm{Ce}_{x} \mathrm{CuO}_{4-y}$ taken from Ref. [8] and $\mathrm{La}_{2-x} \mathrm{Sr}_{x} \mathrm{CuO}_{4}$ from Ref. [3]. 
and then decreases in both underdoped and overdoped regimes. On the other hand, it has been shown ${ }^{21}$ that AFLRO can be stabilized by the $t^{\prime}$ term for the electrondoped case, which may lead to the charge carrier's localization over a broader range of doping, this is also why superconductivity appears over a narrow range of doping in electron-doped cuprates.

In the CSS fermion-spin theory ${ }^{19}$, the physical electron is decoupled as the dressed charge carrier $h_{i \sigma}=e^{-i \Phi_{i \sigma}} h_{i}$ and spin $S_{i}$. Since the phase factor $\Phi_{i \sigma}$ in the dressed charge carrier is separated from the bare spinon operator, and then it describes a spin cloud ${ }^{19}$. Therefore the dressed charge carrier $h_{i \sigma}$ is a spinless fermion $h_{i}$ (bare charge carrier) incorporated the spin cloud $e^{-i \Phi_{i \sigma}}$ (magnetic flux), thus is a magnetic dressing. In other words, the dressed charge carrier carries some spin messages, i.e., it shares its nontrivial spin environment. It has been shown ${ }^{19}$ that these dressed charge carrier and spin are gauge invariant under a local U(1) gauge transformation, and in this sense, they are real and can be interpreted as the physical excitations. In doped cuprates, the normal-state above the SC transition temperature exhibits a number of anomalous properties which is due to $\mathrm{CSS}^{20}$, while the SC state is characterized by the charge-spin recombination ${ }^{26}$. Based on the CSS fermionspin theory, we ${ }^{28}$ have discussed the charge dynamics of the underdoped cuprates in the normal-state, and show that under temperature $T^{*}$, the magnetic fluctuation is strong enough to lead to a pseudogap. This pseudogap would reduce the charge carrier scattering and thus is responsible for the temperature linear to the nonlinear range in the in-plane resistivity and the crossovers to the insulating-like range in the c-axis resistivity. Furthermore, the temperature $T^{*}$ is doping dependent, and grows monotonously as the doping concentration decreases, and disappear in higher doping ${ }^{28}$. It has been shown ${ }^{28}$ that this pseudogap (then the temperature $T^{*}$ ) is obtained from the charge carrier Green's function in the normal-state by considering the second-order correction due to the spin pair bubble. In the kinetic energy driven $\mathrm{SC}$ mechanism ${ }^{17,18}$, the charge carrier pairing state (then the electron SC-state and SC transition temperature $T_{c}$ ) occurrs directly through the kinetic energy by exchanging spin excitations, and is controlled by both charge carrier gap function and single particle coherent weight. This single particle coherent weight is obtained from the charge carrier quasiparticle diagonal Green's function in the SC-state by considering the second-order correction due to the spin pair bubble, and at the same time is effected by the dressed charge carrier pair gap function (then the charge carrier quasiparticle off-diagonal Green's function), which is shown explicitly in the self-consistent equations (7a) and (7b). Moreover, this dressed charge carrier pairing amplitude (then the $\mathrm{SC}$ order parameter) is proportional to the number of charge carrier quasiparticles (then the superfluid density), and not to the number of electrons as mentioned above, which leads to ${ }^{18}$ that the single particle coherent weight $Z_{F}\left(T_{c}\right)$ resembles that of the superfluid density. In other words, $T^{*}$ is closely related to the spin fluctuation, while $T_{c}$ is self-consistently governed by the single particle coherence and dressed charge carrier pair gap function, this is why there are some differences between $T^{*}$ and $T_{c}$.

In summary, within the framework of the kinetic energy driven the $\mathrm{SC}$ mechanism ${ }^{17}$, we have discussed the charge asymmetry in superconductivity of hole- and electron-doped cuprates based on the $t-t^{\prime}-J$ model. Our results show that for the hole-doped case, superconductivity appears over a wide range of doping, where the maximal SC transition temperature occurs around the optimal doping concentration, and then decreases in both underdoped and overdoped regimes. In analogy to the phase diagram of hole-doped case, superconductivity appears over a narrow range of doping in the electron-doped side, where the SC transition temperature increases sharply with increasing doping in the underdoped regime, and reaches a maximum in the optimal doping, then decreases sharply with increasing doping in the overdoped regime. However, the maximum achievable SC transition temperature in the optimal doping in the electron-doped case is much lower than that of the hole-doped side due to the electron-hole asymmetry. Our these results are in qualitative agreement with the experimental observations.

\section{ACKNOWLEDGMENTS}

The author would like to thank Dr. Huaiming Guo for the helpful discussions. This work was supported by the National Natural Science Foundation of China under Grant Nos. 10125415 and 90403005 , and the Grant from Beijing Normal University.

${ }^{1}$ See, e.g., M.A. Kastner, R.J. Birgeneau, G. Shiran, and Y. Endoh, Rev. Mod. Phys. 70, 897 (1998).

2 J.G. Bednorz and K.A. Müller, Z. Phys. B 64, 189 (1986).

${ }^{3}$ See, e.g., J.L. Tallon, J.W. Loram, J.R. Cooper, C. Panagopoulos, and C. Bernhard, Phys. Rev. B 68, 180501 (2003)

${ }^{4}$ Y. Tokura, H. Takagi, and S. Uchida, Nature 337, 345 (1989).

${ }^{5}$ M.Z. Hasan, Y. Li, D. Qian, Y.-D. Chuang, H. Eisaki, S. Uchida, Y. Kaga, T. Sasagawa, and H. Takagi, condmat/0406654, unpublished.

${ }^{6}$ A. Sawa, M. Kawasaki, H. Takagi, and Y. Tokura, Phys. Rev. B 66, 014531 (2002).

${ }^{7}$ H. Takagi, S. Uchida, and Y. Tokura, Phys. Rev. Lett. 62, 1197 (1989). 
${ }^{8}$ J.L. Peng, E. Maiser, T. Venkatesan, R.L. Greene, and G. Czjzek, Phys. Rev. B 55, R6145 (1997).

${ }^{9}$ See, e.g., C.C. Tsuei and J.R. Kirtley, Rev. Mod. Phys. 72, 969 (2000).

10 J.A. Martindale, S.E. Barrett, K.E. ÓHara, C.P. Slichter, W.C. Lee, and D.M. Ginsberg, Phys. Rev. B 47, 9155 (1993); W.N. Hardy, D.A. Bonn, D.C. Morgan, R. Liang, and K. Zhang, Phys. Rev. Lett. 70, 3999 (1994); D.A. Wollman, D.J. Van Harlingen, W.C. Lee, D.M. Ginsberg, and A.J. Leggett, Phys. Rev. Lett. 71, 2134 (1993).

${ }^{11}$ N.P. Armitage, D.H. Lu, D.L. Feng, C. Kim, A. Damascelli, K.M. Shen, F. Ronning, Z.-X. Shen, Y. Onose, Y. Taguchi, and Y. Tokura, Phys. Rev. Lett. 86, 1126 (2001).

12 C.C. Tsuei and J.R. Kirtley, Phys. Rev. Lett. 85, 182 (2000).

${ }^{13}$ K. Yamada, C.H. Lee, K. Kurahashi, J. Wada, S. Wakimoto, S. Ueki, H. Kimura, Y. Endoh, S. Hosoya, and G. Shirane, Phys. Rev. B 57, 6165 (1998); P. Dai, H.A. Mook, R.D. Hunt, and F. Dog̃an, Phys. Rev. B63, 54525 (2001).

${ }^{14}$ K. Yamada, K. Kurahashi, T. Uefuji, S. Park, S.H. Lee, and Y. Endoh, Phys. Rev. Lett. 90, 137004 (2002).

${ }^{15}$ H.J. Kang, P. Dai, J.W. Lynn, M. Matsuura, J.R. Thompson, S.C. Zhang, D.N. Argyriou, Y. Onose, and Y. Tokura, Nature 423, 522 (2003).

${ }^{16}$ H. Matsui, K. Terashima, T. Sato, T. Takahashi, M. Fujita, and K. Yamada, cond-mat/0411547, unpublished.

17 Shiping Feng, Phys. Rev. B 68, 184501 (2003).

18 Tianxing Ma, Huaiming Guo, and Shiping Feng, Mod. Phys. Lett. B 18, 895 (2004); Shiping Feng and Tianxing Ma, cond-mat/0407310, unpublished.

19 Shiping Feng, Jihong Qin, and Tianxing Ma, J. Phys. Condens. Matter 16, 343 (2004); Shiping Feng, Z.B. Su, and L. Yu, Phys. Rev. B 49, 2368 (1994).

${ }^{20}$ P.W. Anderson, Science 235, 1196 (1987).

${ }^{21}$ M.S. Hybertson, E. Stechel, M. Schuter, and D. Jennison, Phys. Rev. B 41, 11068 (1990).

22 Tianxing Ma, Huaiming Guo, and Shiping Feng, Phys. Lett. A337, 61 (2005); Bin Liu, Ying Liang, Shiping Feng and Wei Yeu Chen, Phys. Rev. B 69, 224506 (2004).

${ }^{23}$ Z.X. Shen, D.S. Dessau, B.O. Wells, D.M. King, W.E. Spicer, A.J. Arko, D. Marshall, L.W. Lombardo, A. Kapitulnik, P. Dickinson, S. Doniach, J. DiCarlo, T. Loeser, and C.H. Park, Phys. Rev. Lett. 70, 1553 (1993); H. Ding, M.R. Norman, J.C. Campuzano, M. Randeria, A.F. Bellman, T. Yokoya, T. Takahashi, T. Mochiku, and K. Kadowaki, Phys. Rev. B54, R9678 (1996).

${ }^{24}$ G.M. Eliashberg, Sov. Phys. JETP 11, 696 (1960); D.J. Scalapino, J.R. Schrieffer, and J.W. Wilkins, Phys. Rev. 148, 263 (1966).

25 Tianxing Ma, Huaiming Guo, and Shiping Feng, unpublished.

${ }^{26}$ P.W. Anderson, Phys. Rev. Lett. 67, 2092 (1991); Science 288, 480 (2000); Physica C 341-348, 9 (2000); condmat/0108522.

${ }^{27}$ H. Ding, J.R. Engelbrecht, Z. Wang, J.C. Campuzano, S.C. Wang, H.B. Yang, R. Rogan, T. Takahashi, K. Kadowaki, and D.G. Hinks, Phys. Rev. Lett. 87, 227001 (2001).

${ }^{28}$ Shiping Feng, Feng Yuan, and Weiqiang Yu, Eur. Phys. J. B15, 607 (2000); Shiping Feng and Zhongbing Huang, Phys. Lett. A232, 293(1997). 